\title{
CTGF upregulation correlates with MMP-9 level in airway remodeling in a murine model of asthma
}

\author{
Sheng-Chieh Lin ${ }^{1,2,3}$, Hsiu-Chu Chou ${ }^{4}$, Bor-Luen Chiang ${ }^{3}$, Chung-Ming Chen ${ }^{2,5}$
}

\author{
${ }^{1}$ Department of Pediatrics, Shuang-Ho Hospital, Taipei Medical University, Taipei, \\ Taiwan \\ ${ }^{2}$ Department of Pediatrics, School of Medicine, College of Medicine, Taipei Medical \\ University, Taipei, Taiwan \\ ${ }^{3}$ Graduate Institute of Clinical Medicine, College of Medicine, National Taiwan \\ University, Taipei, Taiwan \\ ${ }^{4}$ Department of Anatomy and Cell Biology, School of Medicine, College of Medicine, \\ Taipei Medical University, Taipei, Taiwan \\ ${ }^{5}$ Department of Pediatrics, Taipei Medical University Hospital, Taipei, Taiwan
}

Submitted: 22 January 2015

Accepted: 5 May 2015

Arch Med Sci 2017; 13, 3: 670-676

DOI: 10.5114/aoms.2016.60371

Copyright () 2016 Termedia \& Banach

\section{Abstract}

Introduction: Connective tissue growth factor (CTGF) mediates hypertrophy, proliferation, and extracellular matrix synthesis. Matrix metalloproteinase (MMP) plays a role in airway extracellular matrix remodeling. The correlation between CTGF and MMP in airway remodeling of asthma was unknown. This study investigated lung CTGF expression and its correlation with MMP and airway structural changes in a murine model of asthma.

Material and methods: Female BALB/c mice were sensitized and challenged by intraperitoneal injections and intranasal phosphate-buffered saline (PBS) or ovalbumin (OVA). Airway responsiveness and serum OVA-specific IgE were measured. Airway structural changes were quantified by morphometric analysis. Differential cell counts and MMP-2, MMP-9, and tissue inhibitor of metalloproteinase (TIMP)-1 were evaluated in bronchoalveolar lavage fluid (BALF). Lung CTGF was determined by Western blot.

Results: Serum OVA-specific IgE level and airway responsiveness in enhanced pause (Penh) is significantly higher in sensitized mice challenged with OVA compared to PBS-challenged mice. MMP-2, MMP-9, and TIMP-1 in BALF were significantly higher in OVA mice. Airway structural changes of animals' lungs with OVA challenge showed increased thickness of the smooth muscle layer and numbers of Goblet cells and inflammatory cells and eosinophils near airways and perivascular areas. Lung CTGF expression significantly increased in OVA-challenged mice. CTGF expressions positively correlated with MMP-9 $(r=0.677, p<0.05)$, TIMP-1 $(r=0.574, p<0.05)$ and thickness of the smooth muscle layer $(r=0.499, p<0.05)$.

Conclusions: This study indicates that CTGF upregulation correlates with MMP-9, probably involved in the pathogenesis of airway remodeling of asthma.

Key words: asthma, airway remodeling, connective tissue growth factor, matrix metalloproteinase.

\section{Introduction}

Asthma is a common disease of children and is one of the most common causes of chronic cough in adult non-smokers, characterized by the

\author{
Corresponding author: \\ Prof. Chung-Ming Chen \\ Department of Pediatrics \\ Taipei Medical University \\ Hospital \\ Taipei 110, Taiwan \\ Phone: +886227372181 \\ E-mail: cmchen@tmu.edu.tw
}


presence of increased numbers of activated mast cells and eosinophils in the airway [1, 2]. In addition to the presence of inflammatory cells in the airway, the airways demonstrate varying levels of structural changes termed airway remodeling $[3,4]$. Structural changes of airway remodeling include elevated numbers of inflammatory cells, deposition of collagen and fibronectin in the subepithelial basement membrane, hyperplasia and hypertrophy of airway smooth muscle, and hypertrophy of submucosal glands $[5,6]$.

Connective tissue growth factor (CTGF) is part of the CTGF-Cyr61/Cef10-Nov family, enabling functions including migration, cell adhesion, hypertrophy, proliferation, and extracellular matrix synthesis [7]. The airway smooth muscle cells participate actively in the inflammatory and the remodeling processes of asthma [8]. Airway smooth muscle cells derived from asthmatic subjects proliferate faster, release a different profile of matrix proteins (fibronectin and collagen I), and produce more CTGF subsequent to transforming growth factor (TGF)- $\beta 1$ stimulation [9-11]. In contrast, Balzar et al. [12] found decreased CTGF expression in endobronchial biopsy tissue and percentages of smooth muscle area of all asthma severity patients. CTGF functions as a downstream mediator of TGF- $\beta 1$. TGF- $\beta 1$ has been suggested to play a critical role in the development of airway remodeling induced by ovalbumin (OVA) [13-16]. However, anti-TGF- $\beta$ treatment had no effect on the development of airway remodeling and exacerbated the eosinophilic infiltrate and led to increased airway hyperreactivity in house dust mite-induced allergic disease [17]. The exact expression of CTGF in airway remodeling is unknown.

Matrix metalloproteinases (MMPs) belong to a family of extracellular proteases that are responsible for the degradation of the extracellular matrix during tissue remodeling [18]. Currently, no effective therapy is clinically available to prevent or reverse airway remodeling in asthma. Therefore, it is critical to understand the pathophysiological events which underlie its mechanism. We hypothesized that structural airway remodeling is associated with increased CTGF expression in a murine model of asthma. The aims of this study were to investigate lung CTGF expression and the relationship of CTGF and MMPs and airway structural changes in a murine model of asthma.

\section{Material and methods}

\section{Animal models}

Six- to eight-week-old female BALB/c mice were purchased from BioLASCO Taiwan Co., Ltd. and were maintained in a pathogen-free facility. Animals were kept at approximately $25^{\circ} \mathrm{C}$ and pelleted food and water were available ad libitum throughout the experiment. The study protocol was approved by the Institutional Animal Care and Use Committee of Taipei Medical University. Eighteen mice were randomly divided into an OVA-challenged group and a phosphate-buffered saline (PBS)-challenged group. Mice $(n=8)$ in the OVA group were sensitized by intraperitoneal injection of $50 \mu \mathrm{g}$ of grade $\mathrm{V}$ chicken egg ovalbumin (OVA, Sigma Chemical Co., St. Louis, MO, USA) and $4 \mathrm{mg}$ of aluminium hydroxide (Imject Alum; Pierce, Rockford, IL, USA) in $0.2 \mathrm{ml}$ of PBS on days 0,14 , and 28 . Mice then were intranasally challenged with OVA on days 40, 41, and 42. Animals were lightly anesthetized using inhaled $2 \%$ isoflurane (Halocarbon Laboratories, River Edge, NJ, USA), and $100 \mu \mathrm{g}$ of OVA in a total volume of $40 \mu \mathrm{l}$ of PBS was intranasally delivered dropwise to the nares using a Pipetman (model P200, Gilson, USA) while the mouse was in an erect position. Control mice $(n=10)$ were sensitized with $4 \mathrm{mg}$ of aluminium hydroxide in $0.2 \mathrm{ml}$ of PBS and intranasally challenged with $40 \mu \mathrm{l}$ of aerosolized PBS solution.

\section{Airway responsiveness measurement}

Airway responsiveness was measured by a technician who was blinded to the protocol and experimental groups using barometric wholebody plethysmography to calculate enhanced pause (Penh: Buxco Electronics Inc., Sharon, CT, USA) on day 43. Mice were exposed to the inhalation of PBS and subsequent increasing doses of methacholine (Sigma, St Louis, MO, USA, 6.25, $12.5,25$, and $50 \mathrm{mg} / \mathrm{ml}$ ). Each nebulization lasted for $3 \mathrm{~min}$ and records were taken for $3 \mathrm{~min}$ after nebulization. Every aerosol was separated by a 15-min recovery period in order to allow airway Penh to return to the baseline level. Data presented in this report correspond to the mean of the first 3-min record performed after each exposure to methacholine or placebo. Penh results were expressed as absolute values.

\section{Serum OVA-specific IgE}

The mice were killed 1 day after airway responsiveness measurement using intraperitoneal injections of pentobarbital $(100 \mathrm{mg} / \mathrm{kg}$, Sigma, St Louis, MO, USA). Serum was obtained through cardiac puncture of anesthetized mice and was stored at $-20^{\circ} \mathrm{C}$ for OVA-specific IgE measurement. OVA-specific IgE levels in serum were measured using an ELISA kit (BioLegend, San Diego, CA, USA).

\section{Evaluation of bronchoalveolar lavage fluid (BALF)}

Immediately after cardiac puncture, the lung was removed with the tracheostomy tube in place. 
The lungs were instilled with $1 \mathrm{ml}$ of ice-cold saline, which was washed in and out of the lungs three times and then recovered. This washing procedure was repeated two more times before recording the total volume of the three washes. Differential cell counts of BALF were performed on cytocentrifuge preparations (Cytospin 3; Shandon Scientific, Cheshire, UK) stained with Liu's stain (Tonyar, Diagnostic Inc., Taiwan) using standard morphologic criteria. The amount of MMP-2, MMP-9, and tissue inhibitor of metalloproteinase (TIMP)- 1 in the BALF was evaluated by ELISA in accordance with the manufacturer's instructions (Cloud-Clone Corp., Houston, USA). The results were expressed in $\mathrm{ng} / \mathrm{ml}$.

\section{Morphometric analysis}

Seven- $\mu \mathrm{m}$ lung sections were embedded in paraffin and stained with hematoxylin and eosin. A pathologist (HCC) who was blinded to the treatment group analyzed three bronchi measuring 150-350 $\mu \mathrm{m}$ in luminal diameter per mouse for the thickness of the epithelium and smooth mus cle layers. Peribronchial inflammation was quantified by counting the number of inflammatory cells and eosinophils surrounding airways and normalizing for airway size by dividing the square of the perimeter of the basement membrane [19].

\section{Western blot analysis}

Lung tissues were homogenized in ice-cold buffer containing $50 \mathrm{mM}$ of Tris $\cdot \mathrm{HCl}(\mathrm{pH} 7.5), 1 \mathrm{mM}$ of EGTA, $1 \mathrm{mM}$ of EDTA, and a protease inhibitor cocktail (complete mini-tablets, Roche, Mannheim, Germany). Proteins (30 $\mu \mathrm{g}$ ) were resolved on 12\% SDS-PAGE under reducing conditions and electroblotted to a polyvinylidene difluoride membrane (ImmobilonP, Millipore, Bedford, MA, USA). After being blocked with 5\% non-fat dry milk, the membranes were incubated with anti-CTGF (1:2000, Abcam, Cambridge, UK) or anti- $\beta$-actin $(1: 20,000$,

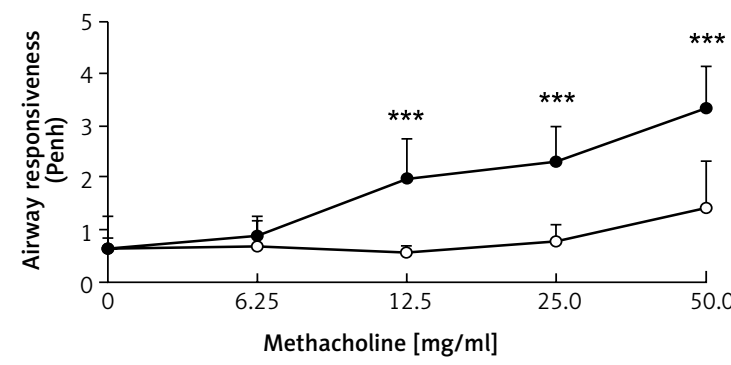

Figure 1. Enhanced pause (Penh) as a function of methacholine concentration. Values are means \pm SD. ○, PBS-challenged mice; $\bullet$, OVA-challenged mice. Penh is significantly higher in sensitized mice challenged with OVA compared to PBS-challenged mice at methacholine concentrations of $12.5 \mathrm{mg} /$ $\mathrm{ml}$ and above $\left({ }^{* * *} p<0.001\right)$
Sigma-Aldrich, St. Louis, MO, USA) and then incubated with horseradish peroxidase-conjugated goat anti-mouse IgG (Pierce Biotechnology, Rockford, USA). Protein bands were detected using SuperSignal Substrate from Pierce. Densitometric analysis was performed to measure the intensity of CTGF and $\beta$-actin bands using AIDA software.

\section{Statistical analysis}

Results are presented as the means \pm SD. Comparisons between control and sensitized-challenged groups were made by Student's $t$-test. The Pearson test was used for correlation analyses of relationships between CTGF and MMP expression and airway structural changes. Differences were considered significant at $p<0.05$.

\section{Results}

\section{Airway responsiveness (Penh)}

Figure 1 shows the airway response in enhanced pause (Penh) vs. methacholine concentration. Penh is significantly higher in sensitized mice with OVA than in PBS mice at challenged methacholine concentrations of $12.5 \mathrm{mg} / \mathrm{ml}$ and above.

\section{OVA-specific IgE}

The OVA-specific IgE levels in serum were significantly elevated $\sim 11$-fold in sensitized mice with OVA compared to PBS mice (Figure 2).

\section{Inflammatory cell infiltrate in BALF}

The differential cell count of BALF revealed a significantly increased percentage of eosinophils, neutrophils, and lymphocytes in the OVA mice compared to PBS mice (Table I). Bronchoalveolar lavage differential cells in OVA mice showed $3.5 \pm 2.9 \%$ eosinophils, $77.7 \pm 10.5 \%$ neutrophils, $12.6 \pm 1.6 \%$ lymphocytes, and $6.2 \pm 6.9 \%$ macrophages, and in PBS mice showed $0 \%$ eosinophils, $27.4 \pm 24.6 \%$ neutrophils, $59.9 \pm 28.6 \%$ lymphocytes, and $12.8 \pm 16.7 \%$ macrophages.

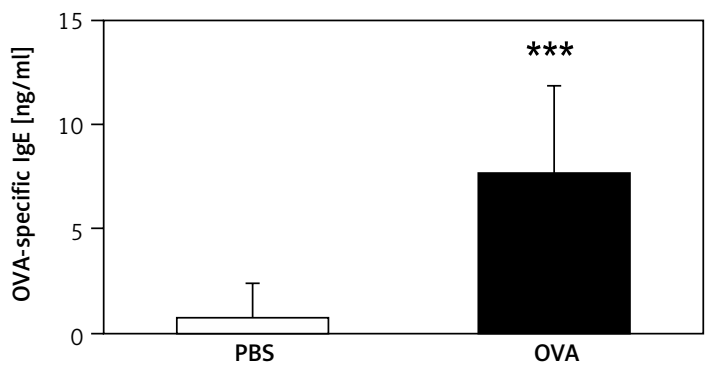

Figure 2. Mouse serum levels of OVA-specific IgE. Serum OVA-specific IgE levels increased significantly in mice sensitized and challenged with OVA ${ }^{* \star *} p<0.001$ vs. saline group) 
Table I. Bronchoalveolar lavage differential cell counts

\begin{tabular}{|llcccc|}
\hline Treatment & $n$ & Eosinophils (\%) & Neutrophils (\%) & Lymphocytes (\%) & Macrophages (\%) \\
\hline PBS & 7 & $0.0 \pm 0.0$ & $27.4 \pm 24.6$ & $59.9 \pm 28.6$ & $12.8 \pm 16.7$ \\
\hline OVA & 8 & $3.5 \pm 2.9^{\star *}$ & $77.7 \pm 10.5^{\star * *}$ & $12.6 \pm 1.6^{* *}$ & $6.2 \pm 6.9$ \\
\hline
\end{tabular}

Differential cell counts of bronchoalveolar lavage fluid were performed on cytocentrifuge preparations using standard morphologic criteria. The results are expressed as the percentage of total cells. Values are means $\pm S D ;{ }^{* *} p<0.01,{ }^{* * *} p<0.001 \mathrm{vs}$. saline group.

\section{Lung histology and morphometric analysis}

The animals' lungs that were sensitized and challenged with OVA and methacholine contained large numbers of inflammatory cells concentrat-

A

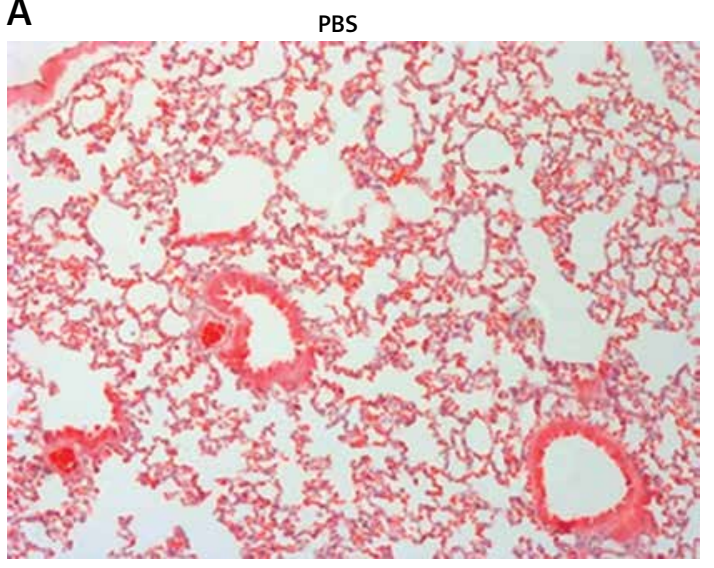

B
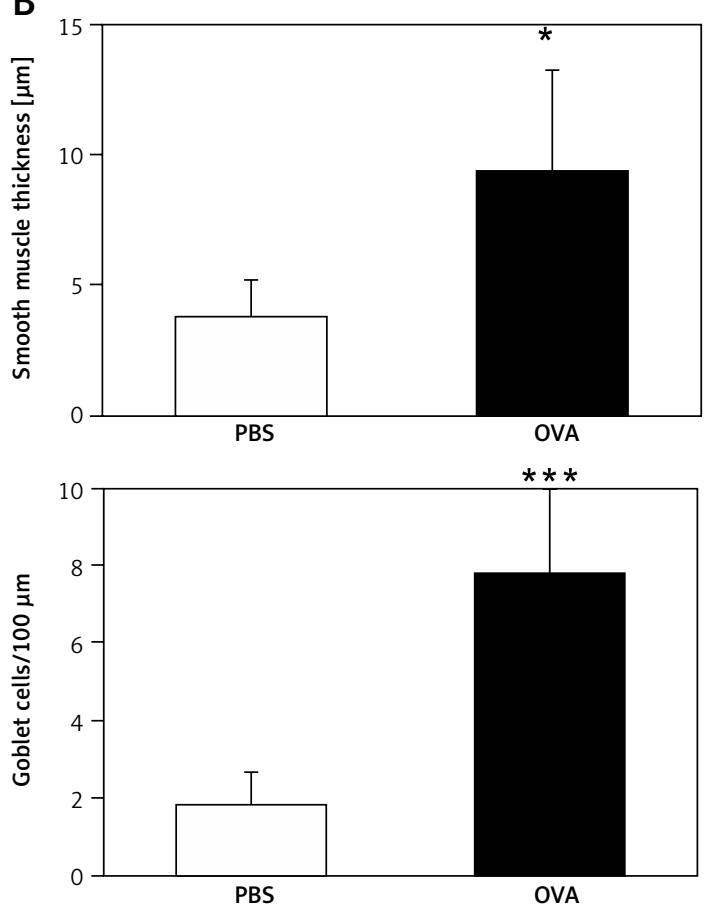

PBS ed near the airways and in the perivascular areas (Figure $3 \mathrm{~A}$ ). The thickness of the smooth muscle layer and the numbers of total inflammatory cells, eosinophils, and goblet cells significantly increased in OVA mice (Figure $3 \mathrm{~B}$ ).
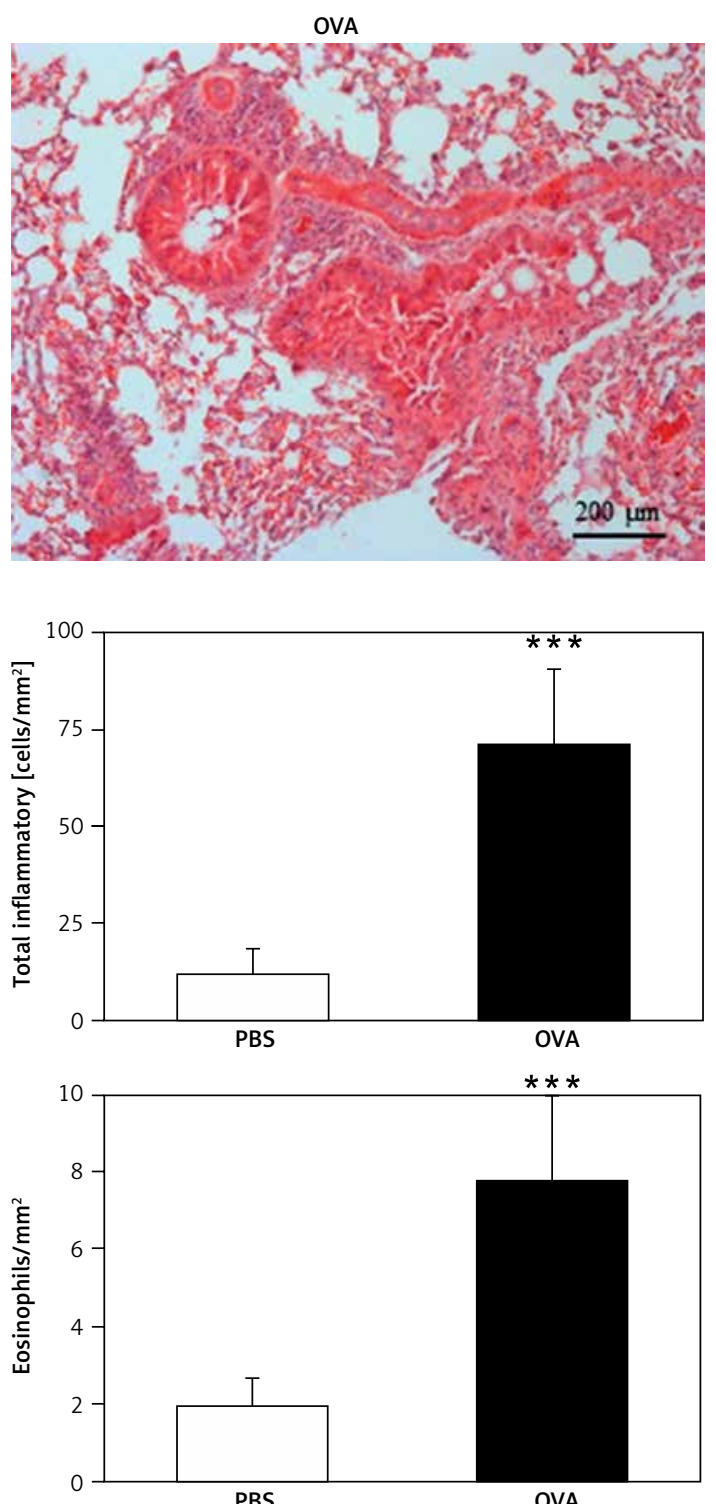

Figure 3. Representative photomicrographs (100x) (A) and morphometric analysis of structural changes (B) and inflammatory cell counts in the airway wall of PBS- and OVA-challenged mice. Three bronchi measuring 150-350 $\mu \mathrm{m}$ in luminal diameter per mouse were analyzed for the thickness of the epithelium and smooth muscle layers and total inflammatory cells and eosinophils surrounding the airways. A - The lungs of animals that were sensitized and challenged with OVA contained large numbers of inflammatory cells concentrated near the airways and in the perivascular areas. B - The thickness of the bronchial epithelium and smooth muscle layer and the numbers of total inflammatory cells and eosinophils increased significantly in OVA-challenged mice $\left({ }^{\star} p<0.05,{ }^{* * *} p<0.001\right.$ vs. PBS group) 


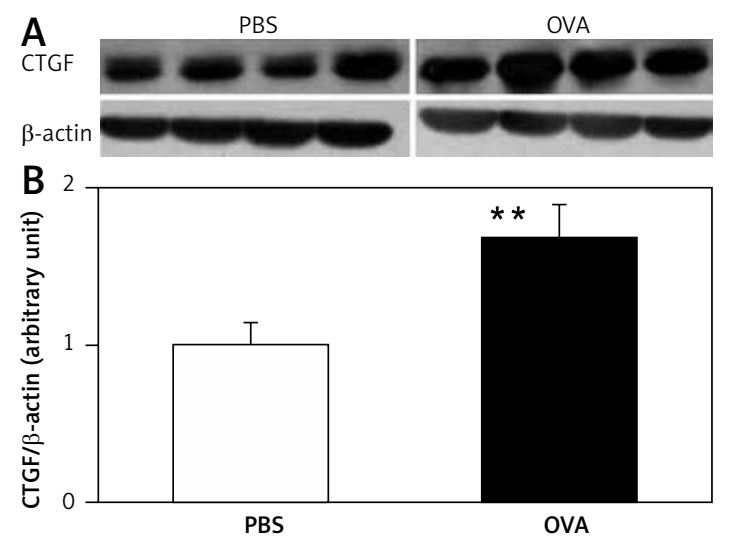

Figure 4. Representative western blotting and scanning densitometry results of the CTGF protein. A The expression of the CTGF protein was higher in the OVA-challenged mice. B - The protein expression levels were normalized to $\beta$-actin for each animal. Data were reported as the fold-change relative to PBS-challenged mice. The level of CTGF expression was significantly higher in the OVA-challenged mice compared to PBS-challenged mice $\left({ }^{* *} p<0.01\right)$

\section{Western blot analysis of CTGF}

Mice sensitized and challenged with OVA and methacholine had significantly higher CTGF protein expression in lung tissues than the mice sensitized and challenged with PBS (Figure 4).

\section{Concentrations of MMP-2, MMP-9, and TIMP-1 in BALF}

Mice sensitized and challenged with OVA had significantly higher concentrations of MMP-2, MMP-9, and TIMP-1 in BALF than the mice sensitized and challenged with PBS (Figure 5).

Correlation between CTGF, MMP, TIMP, and airway structural changes

Lung CTGF protein expression was positively correlated with BALF MMP-9 $(r=0.677, p<0.05)$ and TIMP-1 $(r=0.574, p<0.05)$ in all study animals. Lung CTGF protein expression was also positively correlated with airway structural changes including thickness of the smooth muscle layer $(r=0.499, p<0.05)$, numbers of total inflammatory cells $(r=0.846, p<0.01)$ and eosinophils $(r=0.657, p<0.01)$. There was no significant correlation between CTGF and MMP-2 and numbers of goblet cells.

\section{Discussion}

Airway remodeling refers to structural changes in airways of asthma patients, which include elevated numbers of inflammatory cells, deposition of collagen and fibronectin in the subepithelial basement membrane, hyperplasia and hypertrophy of airway smooth muscle, and hypertrophy of
A

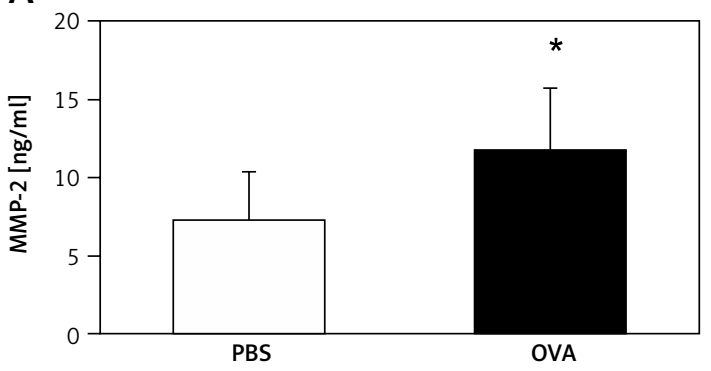

B

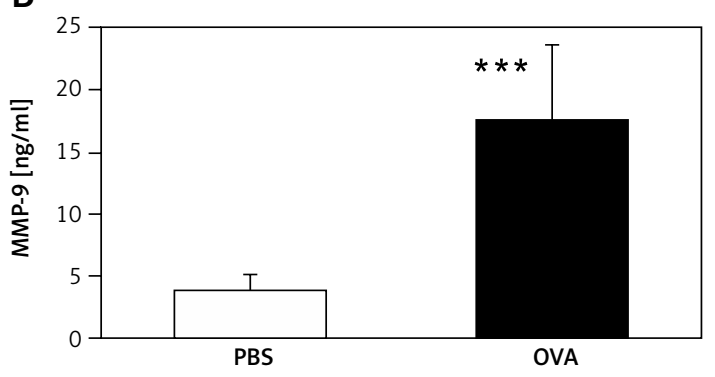

C

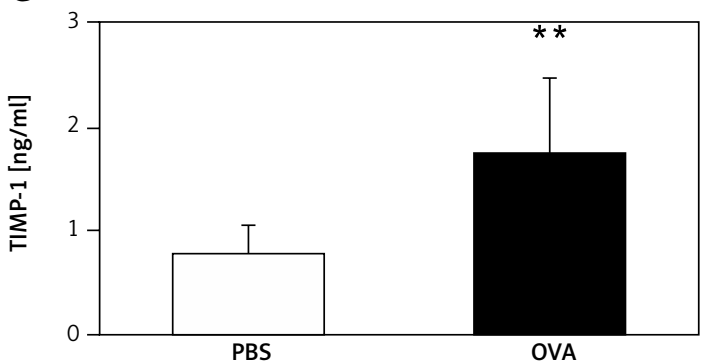

Figure 5. Concentrations of (A) MMP-2, (B) MMP-9, and (C) TIMP-1 in bronchoalveolar lavage fluid $\left({ }^{*} p<0.05,{ }^{\star *} p<0.01,{ }^{* *} p<0.001\right.$ vs. PBS group)

submucosal glands $[5,6]$. Our in vivo animal model showed that sensitization and challenge of mice with OVA induced asthma as demonstrated by the increased airway response in enhanced pause, high serum OVA-specific IgE levels, and increase percentage of eosinophils and inflammatory cells in BALF. Morphometric analysis also revealed airway remodeling as evidenced by increased smooth muscle layer and the numbers of total inflammatory cells and eosinophils in OVA-challenged mice. Large numbers of inflammatory cells were concentrated near the airways and in the perivascular areas. Goblet cells also increased in our murine model of asthma. The experimental finding of this study is that airway remodeling was associated with increased CTGF expression, suggesting that CTGF is involved in the pathogenesis of airway remodeling in asthma.

CTGF, a member of the CCN (CTGF, Cyr61/Cef10, Nov) family, is a cysteine-rich, heparin-binding, 349-amino acid polypeptide [7]. In 1991, CTGF was originally identified in conditioned media from human umbilical vein endothelial cells and mice fibroblasts and was implicated in fibroblast prolif- 
eration, cellular adhesion, angiogenesis, and extracellular matrix synthesis [20]. The TGF- $\beta 1$ has been suggested to play a critical role in the development of airway remodeling induced by ovalbumin (OVA) [13-16]. Eosinophilic infiltrations in inflammatory airways also increased levels of the eosinophil-derived TGF- $\beta$ [21]. Eosinophils are known to play a role in airway remodeling and produce remodeling/proinflammatory cytokines including IL-5, IL-6, IL-11 and IL-17 [22]. This might be a reason why we found that CTGF protein expression in the lungs was significantly positively correlated with total inflammatory cells and eosinophils. In a previous study, CTGF was found to act as a potent downstream mediator of TGF- $\beta 1$ to modulate its functional effects [23]. Therefore, it is conceivable that inhibition of CTGF might block the profibrotic effects without affecting TGF- $\beta$ 's antiproliferative and immunosuppressive effects [24]. In our study, we found that CTGF protein expression significant positively correlated with thickness of the smooth muscle layer of the airway in all study animals. CTGF seems to be a new target in the pathogenesis and progression of airway remodeling. Guo et al. reported that genistein, a soybean and soybased product, inhibited CTGF expression in human renal tubular epithelial cells [25]. Clinically, there is still no effective treatment in children with airway remodeling of asthma. Anti-CTGF might prevent or treat airway remodeling by decreasing the thickness of the airway smooth muscle layer. A better understanding of pathways leading to CTGF upregulation may provide new approaches to treating airway remodeling in asthma.

In this study, we found increased MMP-2, MMP-9, and TIMP-1 levels in BALF in the OVA-challenged mice. Extracellular matrix components have important roles in the thickness of airway smooth muscle [26]. MMP-2 and MMP-9 belong to a family of extracellular proteases that are responsible for the degradation of the extracellular matrix during tissue and vascular remodeling [18, 27]. Levels of MMP-2, MMP-9, and TIMP-1 are significantly increased in BALF from patients and animals with allergic asthma [28-30]. Felsen et al. found that MMP-2 and MMP-9 are up-regulated in the lungs of OVA-challenged mice and the highest MMP-2 and MMP-9 activity was detected in the areas of inflammation surrounding the airways, a major site of tissue remodeling [31]. Matsumoto et al. reported that sputum TIMP levels were positively correlated with airway wall area/body surface area [32]. These studies further suggest that stimulation of bronchial smooth muscle cell migration by matrix metalloproteinases may be an important process in airway remodeling in asthma [33]. We also found that CTGF protein expression in the lungs was significant positively correlated with MMP-9 and TIMP-1 levels in BALF in all study animals. CTGF has been reported to regulate MMP expression [34-36]. Fan et al. [37] reported that CTGF increased MMP expression in vascular smooth muscle cells. These results suggest that increased CTGF expression in combination with increased MMP and TIMP-1 expression could be a cause of the airway remodeling in asthma. This experiment was performed using a small number of animals. Despite this limitation, the study was able to show significant differences between the 2 groups.

In conclusion, this study found increased CTGF expression in a murine model of asthma. These results suggest that CTGF correlates with MMP-9 and TIMP-1 expression, probably involved in the pathogenesis of airway remodeling, and anti-CTGF might prevent or treat airway remodeling.

\section{Acknowledgments}

This work was supported by the Ministry of Science and Technology (MOST 103-2314-B-038019-MY3).

\section{Conflict of interest}

The authors declare no conflict of interest.

\section{References}

1. Cohn L, Elias JA, Chupp GL. Asthma: mechanisms of disease persistence and progression. Annu Rev Immunol 2004; 22: 789-815.

2. Dąbrowska M, Grabczak EM, Arcimowicz M, et al. Chronic cough - assessment of treatment efficacy based on two questionnaires. Arch Med Sci 2014; 10: 962-9.

3. Mauad T, Bel EH, Sterk PJ. Asthma therapy and airway remodeling. J Allergy Clin Immunol 2007; 120: 9971009.

4. Boulet LP, Sterk PJ. Airway remodeling: the future. Eur Respir J 2007; 30: 831-4.

5. Royce SG, Lim CX, Patel KP, Wang B, Samuel CS, Tang ML. Intranasally administered serelaxin abrogates airway remodelling and attenuates airway hyperresponsiveness in allergic airways disease. Clin Exp Allergy 2014; 44: 1399-408.

6. Bergeron C, Al-Ramli W, Hamid Q. Remodeling in asthma. Proc Am Thorac Soc 2009; 6: 301-5.

7. Moussad EE, Brigstock DR. Connective tissue growth factor: what's in a name? Mol Genet Metab 2000; 71: 276-92.

8. Doeing DC, Solway J. Airway smooth muscle in the pathophysiology and treatment of asthma. J Appl Physiol (1985) 2013; 114: 834-43.

9. Burgess JK, Johnson PR, Ge Q, et al. Expression of connective tissue growth factor in asthmatic airway smooth muscle cells. Am J Respir Crit Care Med 2003; 167: 71-7.

10. Black JL, Burgess JK, Johnson PR. Airway smooth muscle: its relationship to the extracellular matrix. Respir Physiol Neurobiol 2003; 137: 339-436.

11. Johnson PR, Burgess JK, Ge Q, et al. Connective tissue growth factor induces extracellular matrix in asthmatic airway smooth muscle. Am J Respir Crit Care Med 2006; 173: 32-41. 
12. Balzar S, Chu HW, Silkoff P, et al. Increased TGF- $\beta 2$ in severe asthma with eosinophilia. J Allergy Clin Immunol 2005; 115: 110-7.

13. McMillan SJ, Xanthou G, Lloyd CM. Manipulation of allergen-induced airway remodeling by treatment with anti-TGF-beta antibody: effect on the Smad signaling pathway. J Immunol 2005; 174: 5774-80.

14. Le AV, Cho JY, Miller M, McElwain S, Golgotiu K, Broide $\mathrm{DH}$. Inhibition of allergen-induced airway remodeling in Smad 3-deficient mice. J Immunol 2007; 178: 7310-6.

15. Alcorn JF, Rinaldi LM, Jaffe EF, et al. Transforming growth factor-beta1 suppresses airway hyperresponsiveness in allergic airway disease. Am J Respir Crit Care Med 2007; 176: 974-82.

16. Chen $M$, Lv Z, Jiang S. The effects of triptolide on airway remodelling and transforming growth factor-beta1/ Smad signaling pathway in ovalbumin-sensitized mice. Immunology 2011; 132: 376-84.

17. Fattouh R, Midence NG, Arias K, et al. Transforming growth factor-beta regulates house dust mite-induced allergic airway inflammation but not airway remodeling. Am J Respir Crit Care Med 2008; 177: 593-603.

18. Lu P, Takai K, Weaver VM, Werb Z. Extracellular matrix degradation and remodeling in development and disease. Cold Spring Harbor Perspect Biol 2011; 3: a005058.

19. Chen CM, Wu MY, Chou HC, Lang YD, Wang LF. Downregulation of caveolin-1 in a murine model of acute allergic airway disease. Pediatr Neonatol 2011; 52: 5-10.

20. Bradham DM, Igarashi A, Potter RL, Grotendorst GR. Connective tissue growth factor: a cysteine-rich mitogen secreted by human vascular endothelial cells is related to the SRC-induced immediate early gene product CEF-10. J Cell Biol 1991; 114: 1285-94.

21. Broide DH. Immunologic and inflammatory mechanisms that drive asthma progression to remodeling. J Allergy Clin Immunol 2008; 121: 560-70.

22. Halwani R, Al-Muhsen S, Hamid Q. Airway remodeling in asthma. Curr Opin Pharmacology 2010; 10: 236-45.

23. Grotendorst GR, Okochi H, Hayashi N. A novel transforming growth factor beta response element controls the expression of the connective tissue growth factor gene. Cell Growth Differ 1996; 7: 469-80.

24. Blom IE, Goldschmeding R Leask A. Gene regulation of connective tissue growth factor: new target for antifibrotic therapy? Matrix Biol 2002; 21: 473-82.

25. Guo Y, Zhang A, Ding Y, Wang Y, Yuan W. Genistein ameliorates parathyroid hormone-induced epithelialto-mesenchymal transition and inhibits expression of connective tissue growth factor in human renal proximal tubular cells. Arch Med Sci 2013; 9: 724-30.

26. An SS, Bai TR, Bates JH, et al. Airway dynamics: a common pathway of airway asthma. Eur Respir J 2007; 29: 834-60.

27. Vitlianova K, Georgieva J, Milanova M, Tzonev S. Blood pressure control predicts plasma matrix metalloproteinase- 9 in diabetes mellitus type II. Arch Med Sci 2015 11: $85-91$.

28. Mautino G, Oliver N, Chanez P, Bousquet J, Capony F. Increased release of matrix metalloproteinase- 9 in bronchoalveolar lavage fluid and by alveolar macrophages of asthmatics. Am J Respir Cell Mol Biol 1997; 17: 583-91.

29. Kim JS, Kang JY, Ha JH, et al. Expression of nerve growth factor and matrix metallopeptidase-9/tissue inhibitor of metalloproteinase- 1 in asthmatic patients. J Asthma 2013; 50: 712-7.

30. Uenolio T, Shibakura M, lio K, et al. Effect of fudosteine, a cysteine derivative, on airway hyperresponsiveness, inflammation, and remodeling in a murine model of asthma. Life Sci 2013; 92: 1015-23.

31. Felsen CN, Savariar EN, Whitney M, Tsien RY. Detection and monitoring of localized matrix metalloproteinase upregulation in a murine model of asthma. Am J Physiol Lung Cell Mol Physiol 2014; 306: L764-74.

32. Matsumoto H, Niimi A, Takemura M, et al. Relationship of airway wall thickening to an imbalance between matrix metalloproteinase- 9 and its inhibitor in asthma. Thorax 2005; 60: 277-81.

33. Nishihara-Fujihara $M$, Shoji $S$, Maeda I, Shimoda $T$, Nishima S, Okamoto K. Involvement of fibronectin and matrix metalloproteinases in airway smooth muscle cell migration for the process of airway remodeling. Allergol Int 2010; 59: 267-75.

34. Blaney Davidson EN, Vitters EL, Mooren FM, Oliver N, Berg WB, van der Kraan PM. Connective tissue growth factor/CCN2 overexpression in mouse synovial lining results in transient fibrosis and cartilage damage. Arthritis Rheum 2006; 54: 1653-61.

35. Maeta N, Osaki M, Shomori K, et al. CYR61 downregulation correlates with tumor progression by promoting MMP-7 expression in human gastric carcinoma. Oncology 2007; 73: 118-26.

36. Chintala H, Liu H, Parmar R, et al. Connective tissue growth factor regulates retinal neovascularization through p53 protein-dependent transactivation of the matrix metalloproteinase (MMP)-2 gene. J Biol Chem 2012; 287: 40570-85.

37. Fan WH, Karnovsky MJ. Increased MMP-2 expression in connective tissue growth factor over-expression vascular smooth muscle cells. J Biol Chem 2002; 277: 9800-5. 